\title{
Design and construction of a localized surface plasmon resonance-based gold nanobiosensor for rapid detection of brucellosis
}

\author{
Sina Vakili ${ }^{1,2}$, Gholamreza Asadikaram ${ }^{3, *}$, Masoud Torkzadeh-Mahani ${ }^{4}$, Alimohammad \\ Behroozikhah $^{5}$, Mohammad Hadi Nematollahi ${ }^{6}$, Amir Savardashtaki ${ }^{7}$ \\ ${ }^{1}$ Neuroscience Research Center, Institute of Neuropharmacology, Kerman University of Medical \\ Sciences, Kerman, Iran. \\ ${ }^{2}$ Infertility Research Center, Shiraz University of Medical Sciences, Shiraz, Iran. \\ ${ }^{3}$ Endocrinology and Metabolism Research Center, Institute of Basic and Clinical Physiology \\ Sciences, Kerman University of Medical Sciences, Kerman, Iran. \\ ${ }^{4}$ Dept. of Biotechnology, Institute of Science, High Technology and Environmental Sciences, \\ Graduate University of Advanced Technology, Kerman, Iran. \\ ${ }^{5}$ Department of Brucellosis, Razi Vaccine and Serum Research Institute. Agricultural, Research, \\ Education and Extension Organization (AREEO), Karaj, Iran. \\ ${ }^{6}$ Herbal and Traditional Medicines Research Center, Kerman University of Medical Sciences, \\ Kerman, Iran. \\ ${ }^{7}$ Dept. of Medical Biotechnology, School of Advanced Medical Sciences and Technology, \\ Shiraz University of Medical Sciences, Shiraz, Iran. \\ Corresponding authors: Gh_asadi@kmu.ac.ir
}

\begin{abstract}
In this study, a localized surface plasmon resonance (LSPR) nanobiosensor was designed to quantify anti-Brucella antibodies in the human sera. Smooth Lipopolysaccharide (LPS) was extracted from Brucella melitensis via a modified hot phenol water method and fixed on the surface of the gold nanoparticles by covalent interactions with the functionalized nanoparticles. To obtain the best performance from the designed probe, the ratio of LPS to gold nanoparticle was optimized. Dynamic light scattering was used for the characterization of the probe. The reduction of the LSPR peak at $600_{\mathrm{nm}}$ was used to quantify the amount of captured anti-Brucella antibody. Finally, satisfactory results were obtained when the nanobiosensor was used to analyze the control and patient sera for the presence of anti-Brucella antibodies.
\end{abstract}

Keywords: Brucella melitensis; brucellosis; gold nanoparticles; LSPR; nanobiosensor.

\section{Introduction}

Biosensors are constructed from a biologic part that can specifically recognize a substance and a physical transduction unit which converts the biochemical interaction to a measurable signal (Sepulveda et al., 2009). Recent advances in nanotechnology greatly contributed to the development of nanoparticle-based optical biosensors (Bellan et al., 2011), and surface plasmon resonance-based biosensors are among the most utilized optical biosensors (Faridli et al., 2016). Nanomaterials are used for different applications owing to their excellent surface area (Mirabi et al., 2019). The attractive optical characteristics of metal nanoparticles are due to their localized surface plasmon resonance (LSPR). LSPR is an optical phenomenon induced by free electrons fluctuation in the conductor metal nanoparticle, and optical spectroscopy is the simplest method for its detection (Sepulveda et al., 2009). Based on the origins of the LSPR, two types of sensors can be designed; sensors based on aggregation and sensors based on the refractive index. The latter changes induced by biomolecular interactions can be measured on 
the gold nanoparticle surface as the LSPR peak shifts. The most common materials for plasmonic applications are noble metals, especially silver and gold. Among various types of nanoparticles, gold nanoparticles are the most used nanomaterial because of their special features such as biocompatibility, watersolubility, non-toxicity, and ease of attachment to biomolecules (Sepulveda et al., 2009; Cobley et al., 2011; Hong et al., 2012; Aqeel et al., 2018). Brucellosis is the most prevalent zoonotic disease globally and takes the life of more than a halfmillion persons per year (Araj, 2010) and causes severe health and economic problems (Jay et al., 2018)

The causative agent is gram-negative bacteria in the genius of Brucella. The primary pathogens in humans are Brucella abortus and B. melitensis and can induce acute and chronic infections (Franco et al., 2007). The clinical symptoms of the disease are common and similar to that of many infections, making the diagnosis of Brucellosis difficult. The disease is commonly misdiagnosed and is referred to as "the disease of mistakes" (Araj, 2010). The main diagnostic method of humans is reviewing patient history and epidemiological information of the disease and most importantly, specific laboratory tests (Franco et al., 2007; Almuneef et al., 2004).

Currently, the best way to correctly diagnose the disease is blood culture, serological assays, and molecular techniques (Araj, 2010). The gold standard for diagnosis of Brucellosis is blood culture (Kaden et al., 2017). Although the positive blood culture is considered a conclusive sign of infection, the incubation time is often too long, and the sensitivity is low in chronic cases. Furthermore, because Brucellosis is one of the most common laboratory-acquired diseases, necessary precautions must be considered to protect laboratory personnel (Araj, 2010; Franco et al., 2007). The introduction of new technologies allowed the use of molecular-based techniques such as PCR and real-time PCR to diagnose the disease (Kaden et al., 2017).
However, these methods have a high risk of contamination and are time-consuming, and are not suitable for routine laboratory assays. (Franco et al., 2007; Kattar et al., 2007; Mika et al., 2007).

The most frequent and reliable method for the detection of Brucellosis is serological-based assays. This will allow the detection of antiBrucella antibodies in the patient's sera and other body fluids (Nielsen \& Yu, 2010; Gomez et al., 2008; Araj, 2010). Commonly used serological assays are agglutination tests and ELISA. The antigen used in the agglutination assay is the whole bacterium. In ELISA is LPS from the smooth strains of Brucella, which is the most recognized antigen of the bacteria. Serological assays are subjected to limitations such as variability in agglutination assays and low sensitivity and time-consuming for ELISA (Franco et al., 2007). Thus, despite recent advances, the clinical diagnosis of the disease remains challenging, and often a combination of available assays is used to diagnose the disease (Araj, 2010).

Due to the simplicity, accuracy, and advances in biosensors based on surface plasmon resonance (Faridli et al., 2016), we introduced a refractive index LSPR nanobiosensor to detect anti-Brucella antibodies in biological samples for diagnosis of Brucellosis.

\section{Experimental}

\subsection{Bacterial culture and LPS extraction}

The smooth strain of Brucella melitensis was cultured in Luria-Bertani (LB) agar. Bacteria were harvested, and LPS was extracted using the modified hot phenol water method (Moreno et al., 1979). SDS-PAGE with silver nitrate staining was used to confirm the quality of extracted LPS. LPS quantification was done using 1,9-dimethylmethylene blue using Salmonella typhimurium as a standard. Bradford method and absorbance at $260_{\text {num }}$ were used to assess the protein and nucleic acid contamination, respectively. 


\subsection{Synthesis of gold nanoparticles}

Gold nanoparticles were synthesized using a chemical reduction of gold salt (HAuC14) (Martin et al., 2010). $15 \mathrm{mg}$ of sodium citrate was dissolved in $50 \mathrm{ml}$ of distilled deionized water. The solution was kept in an ice bath and mixed on a magnetic stirrer (150 rpm). While mixing, $600 \mu \mathrm{l}$ of gold salt solution $(17.3 \mathrm{mM})$ was added. Next, $1.2 \mathrm{ml}$ of sodium borohydride solution $(20 \mathrm{mM})$ was added. The solution was mixed for 2 hours and under the same conditions and then kept in $4^{\circ} \mathrm{C}$ for further use. Based on past studies, in this type of synthesis, sodium citrate simultaneously acts as a reducing agent (driving the reduction of AuIII to Au0), capping agent (electrostatically stabilizing the gold nanoparticles colloidal solution), and $\mathrm{pH}$ mediator (modifying the reactivity of $\mathrm{Au}$ species involved in the reaction). In this assay, a red-colored solution from a yellow-colored solution of HAuCl4 indicating the formation of gold in a zero-oxidation state (Leng et al., 2015). Scanning electron microscopy was used to study the morphology of gold nanoparticles, and size distribution was assessed using Zetasizer NanoZS90 (Malvern Instruments Ltd, Malvern, Worcestershire, UK).

\subsection{Construction of nanoprobe (biosensor)}

\subsubsection{Carboxylation of gold nanoparticles}

To prepare the surface of gold nanoparticles for loading LPS, gold nanoparticles were coated with thioglycolic acid (TGA) linkers. Briefly, 1 $\mathrm{ml}$ of gold nanoparticle solution was mixed with $1 \mathrm{ml}$ of TGA solution $(1 \mathrm{mM})$ and kept at room temperature for 24 hours. For isolation of coated gold nanoparticles, the solution was centrifuged at $12000 \mathrm{~g}$ for $15 \mathrm{~min}$. Excess TGA was removed by two washing steps with double-distilled deionized water. Spectrophotometry was used to analyze the coating of TGA on the surface of the gold nanoparticles.

\subsubsection{Optimizing coating of TGA to gold nanoparticles}

To optimize the coating ratio of TGA on gold nanoparticles, the coating was done at different times. Optical density was measured and graphed to obtain the best incubation time.

\subsubsection{Covalent attachment of LPS to TGA - modified gold nanoparticles}

To stimulate the covalent attachment between the amine group of LPS and the carboxyl group of TGA, the carboxyl groups were activated by EDC and NHS molecules. Sedimented gold nanoparticles were suspended in 0.1 $\mathrm{mM}$ EDC/NHS solution and incubated at room temperature for $30 \mathrm{~min}$. Next, $2 \mathrm{ml}$ of PBS containing $0.05 \%$ Tween-20 (PBST) ( $\mathrm{pH}$ 7.4) was added, and the solution was vortexed vigorously. Nanoparticles were then centrifuged at $12000 \mathrm{~g}$ for $15 \mathrm{~min}$. The supernatant was removed, and the LPS solution was added. The mixture was put in an ultrasonic bath for $10 \mathrm{~min}$ and then incubated at room temperature for 3 hours. Then, 2 $\mathrm{ml}$ of PBST was added, and the solution was vigorously vortexed. The solution was centrifuged for $15 \mathrm{~min}$ at $12000 \mathrm{~g}$, and the supernatant was removed. Nanoprobe was then resuspended in $500 \mu 1$ of PBS, and LSPR spectra were measured by spectrophotometer. After confirming LPS attachment to the gold nanoparticles, the solution was kept at $4^{\circ} \mathrm{C}$ for further investigation.

\subsubsection{Optimizing the LPS concentration}

Optimization of the LPS-to-Au nanoparticle ratio for maximum attachment of LPS to activated TGA carboxyl groups was done by the study of the peak shift in LSPR spectra as a function of LPS concentration (100, 150, 200, 300 , and $560 \mu \mathrm{g} / \mathrm{ml}$ ) in a fixed amount of the $\mathrm{Au}$ nanoparticles. 
2.3.5. Detection of anti-LPS antibodies by nanoprobe

Samples were diluted 1:50 in PBS, and $100 \mu 1$ of the diluted samples were mixed with $200 \mu 1$ of the biosensor and mixed by pipetting. After $30 \mathrm{~min}$ of incubation at room temperature, the biosensors were centrifuged at $12000 \mathrm{~g}$ for 15 min. The biosensors were suspended in $200 \mu 1$ of PBS, and the absorbance was measured as before.

\section{Results and discussion}

\subsection{LPS extraction}

The LPS extraction yield was about $1 \%$ of the wet weight used bacteria. The nucleic acid concentration was less than $0.2 \%$ of the LPS concentration. No protein contamination could be detected by the Bradford method.

\subsection{Synthesis of gold nanoparticle}

Acquiring the best response from the biosensor depends on the size distribution of the nanoparticles. To achieve high affinity, high sensitivity, and high selectivity in interaction with biological targets, the size of the nanoparticles should be small enough to yield good colloidal stability, high surface-to-volume ratios, and fast movement for high binding rates and also should be large enough to allow the attendance of various ligands on the surface of the particle to attain multivalent interactions. In the interactions of proteins, the size of the nanoparticles should be comparable to the size of biological targets (Gu et al., 2006). The plasmon bandwidth is also shown to be narrow enough in particles between 8 to $25_{\mathrm{nm}}$ (Mortazavi et al., 2012). Based on the size of anti brucella antibodies (Fornara et al., 2008), gold nanoparticles were prepared with an average size of $10_{\mathrm{nm}}$. A Zetasizer NanoZS90 (Malvern Instruments Ltd, Malvern, Worcestershire, UK) instrument was used to measure the nanoparticles' size distribution. Dynamic light scattering at a scattering angle of $90^{\circ}$ was used as the basic principle for measuring particle size. Zetasizer
Nano uses a laser with a $633_{\mathrm{nm}}$ wavelength. With this technique, the diffusion of particles caused by Brownian motion is measured and converts to size distribution by the StokesEinstein relationship (Salahvarzi et al., 2017). As shown in Figure 1, there is a sharp peak at the $10_{\mathrm{nm}}$ scale, which indicated that the nanoparticles were homogeneously sized at $10_{\mathrm{nm}}$. Moreover, SEM has confirmed the uniformity of the gold nanoparticles, see later. To determine the wavelength that indicates, a spectrophotometer investigated the maximum absorbance by gold nanoparticles, visual and ultraviolet wavelengths. As shown in Figure 1, the maximum absorbance was at $530_{\mathrm{nm}}$.
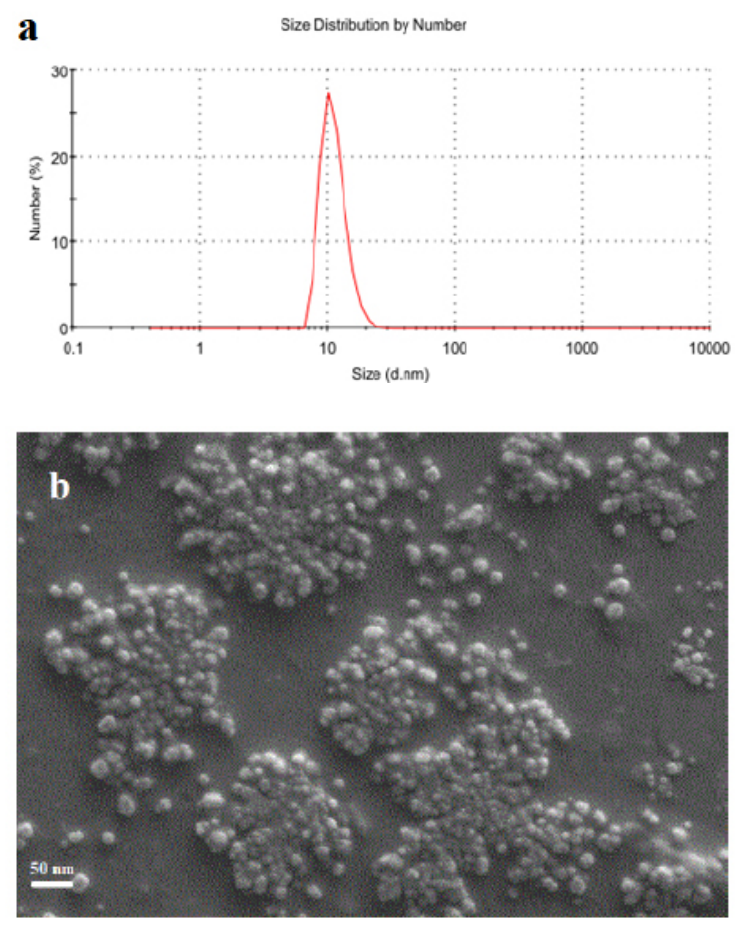

Fig. 1. (a) Size distribution of the synthesized gold nanoparticles by a size analyzer and (b) structure of gold nanoparticles under SEM.

\subsection{Construction of nanoprobe}

\subsubsection{Carboxylation of gold nanoparticles}

TGA molecules were used as a linker between the gold nanoparticles and LPS. After coating the gold nanoparticles with TGA, as depicted in Figure 3, the LSPR peak at $530 \mathrm{~nm}$ was slightly reduced. 


\subsubsection{Optimizing coating of TGA to gold nanoparticles}

The optimization was carried out to determine the best incubation time for maximum TGA adherence to the gold nanoparticles. The results indicated that 24 hours' incubation time yielded in the maximum TGA coating on the surface of the gold nanoparticles.

3.3.3. Covalent attachment of LPS to TGAmodified gold nanoparticles

The LPS was covalently attached to the TGAmodified gold nanoparticles after activating the carboxyl groups of TGA by EDS/NHS solution. Spectrophotometric analysis showed that after LPS attachment, the LSPR peak was shifted from $530_{\mathrm{nm}}$ to $600_{\mathrm{nm}}$. Furthermore, the LSPR of the nanoprobe at $600_{\mathrm{nm}}$ was less than the LSPR at $530_{\mathrm{nm}}$ (Figure 5).

\subsubsection{Optimizing the LPS concentration}

Various concentrations of LPS were used to calculate the maximum attachment of LPS to the gold nanoparticles. Increment in the LPS concentrations resulted in a reduction in the absorbance. However, the LSPR peak remained at $600_{\mathrm{nm}}$. As shown in Figure 6, a concentration of $300 \mu \mathrm{g} / \mathrm{ml}$ was chosen as an optimum concentration for coating the gold nanoparticles.

\subsection{Dtection of anti-LPS antibodies by} nanoprobe

TheLSPR technique can study the interaction of the anti-LPS antibody with lipopolysaccharide antigen in the gold nanoparticles' vicinity as a surface-sensitive optical method. The changes in the LSPR absorption peak are a result of electrostatic interaction between antibody and antigen. As depicted in Figure.7, positive (Anti-Brucella LPS antibodies with a titer of 1:80) and negative controls were assayed for confirming the proper function of the biosensor. The LSPR spectrum of the biosensor was recorded after incubation with positive and negative controls.

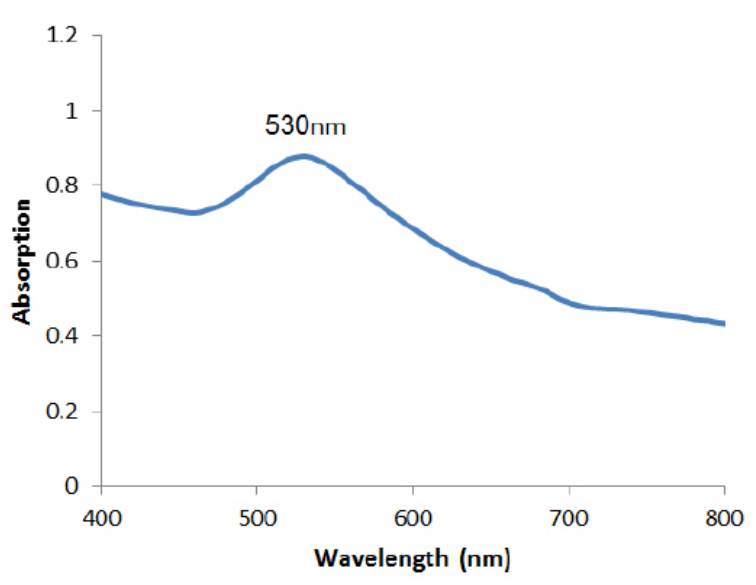

Fig. 2. LSPR peak of synthesized gold nanoparticles.

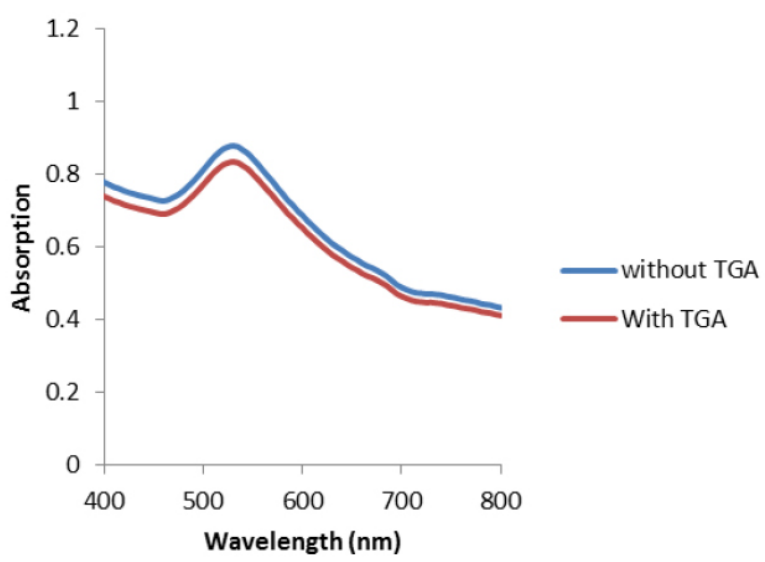

Fig. 3. LSPR peak of gold nanoparticle after coating with TGA.

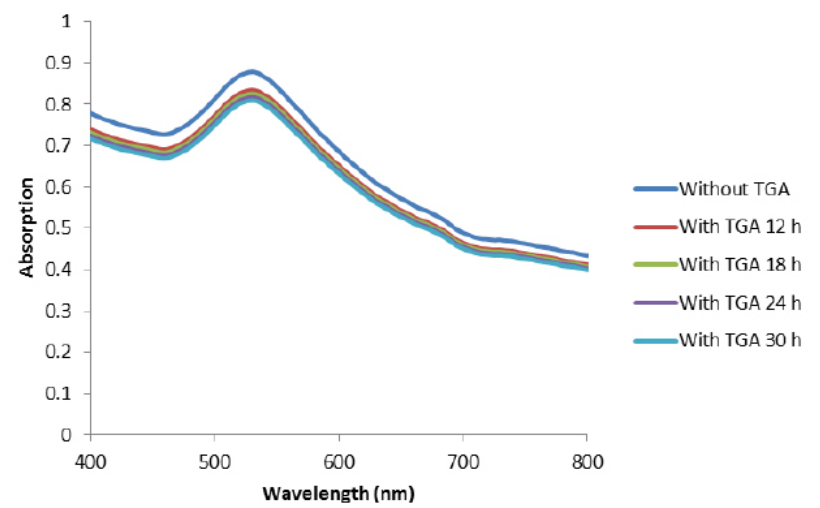

Fig. 4. Optimizing the incubation time of gold nanoparticles with TGA. 
The difference between the intensity of the LSPR peak reduction of the nanoprobe before and after incubation with the negative and positive control was 0.034 and 0.303 , respectively. As expected, the intensity of the LSPR absorption peak did not show significant change after incubation with negative control. On the other hand, the incubation of nanoprobe with positive control resulted in a significant reduction in the LSPR peak at $600_{\mathrm{nm}}$. This showed that the existing anti-LPS antibodies in the positive control have been attached to the related antigen (LPS) and limited the access of light to the surface of nanoprobes. Due to the lack of anti-LPS antibodies in the negative control, the LSPR absorption was not changed (Holzinger et al., 2014). To evaluate the sensitivity and specificity of the designed biosensor and compare the results with the standard laboratory technique for detecting Brucellosis, we tested 4 sera obtained from patients with confirmed Wright test at dilutions $1: 160,1: 320,1: 640$, and 1:1320 and also one serum with confirmed negative results. As expected, as the anti-LPS antibody (positive sera) concentration increased, the LSPR peak at $600_{\mathrm{nm}}$ decreased. However, the analysis of samples did not show a linear relationship between the antibody concentration and reduction in the LSPR peak at $600_{\mathrm{nm}}$. This probably could be explained by saturation of the antibody binding sites on the surface of the nanoprobe, which reduces the access of all antibodies to attach the surface of the nanoprobe. Thus, more studies need to optimize the nanoprobe-sera ratio. Moreover, the reduction of the LSPR peak at $600_{\mathrm{nm}}$ after attachment of antibody was associated with an increase in the LSPR at a shorter wavelength $\left(400-500_{\mathrm{nm}}\right)$ which needs to be investigated (Figure 8).

It should be noted that the current ELISA kits are also unable to quantify the exact amount of anti-LPS antibodies in the sera, and the results are reported as positive or negative based on the obtained values above or below a cut-off point (Padilla et al., 2010). Scientific reports suggest that an antibody titer of 1:80 shows suitable sensitivity and specificity for an ELISA assay and is usually considered a cut-off

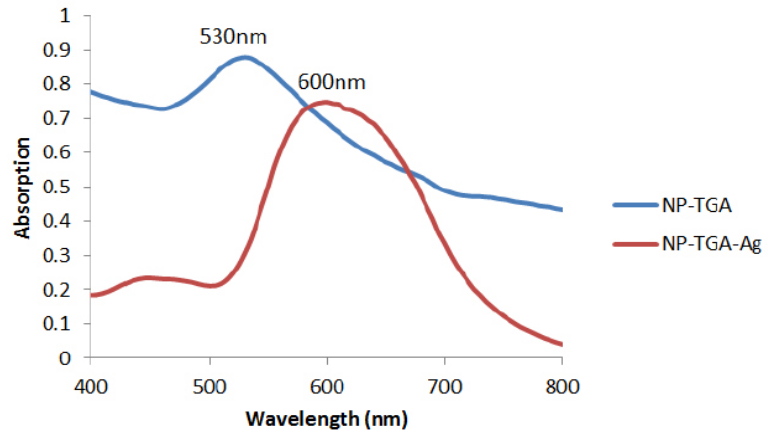

Fig. 5. LSPR peak of nanoprobes.

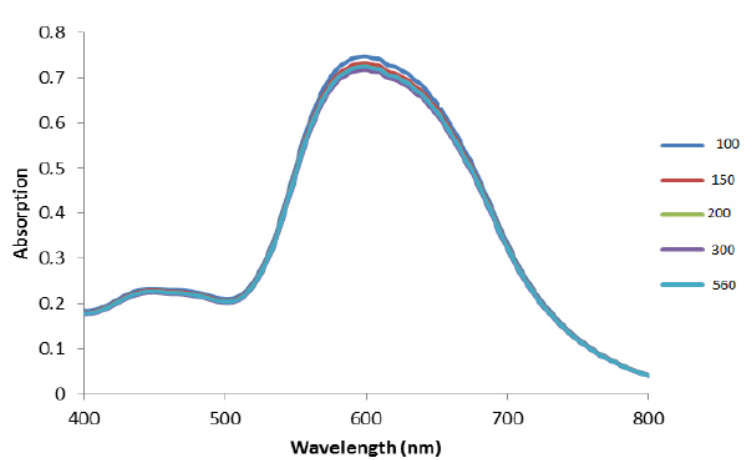

Fig. 6. Optimization of LPS concentration $(\mu \mathrm{g} /$ $\mathrm{ml}$ ) for maximizing LP attachment to the TGA carboxyl groups on the surface of gold nanoparticles.

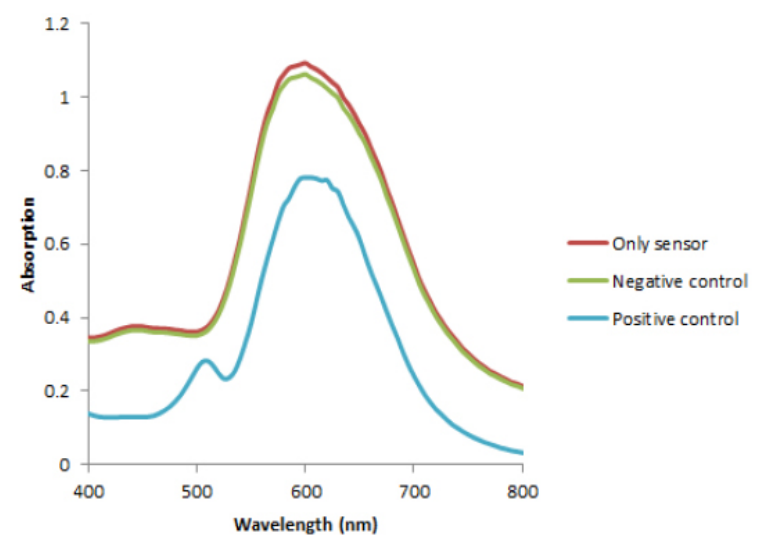

Fig. 7. LSPR peak of nanoprobe in the presence of positive and negative controls

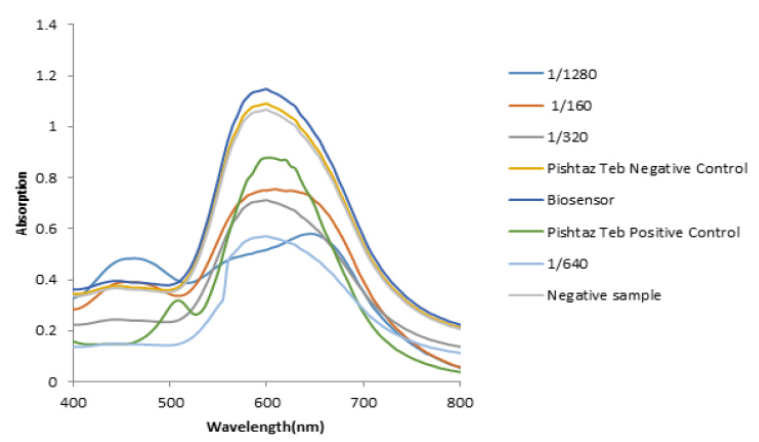

Fig. 8. LSPR peak reduction after incubation with patient sera. 
point. However, establishing the cut-off point depends on the region that the test is being done, as, in endemic regions, higher cut-offs are considered to avoid false-positive results (Franco et al., 2007). According to the results obtained, the designed biosensor shows advantages over the conventional ELISA technique by being more economical, less timeconsuming, and giving more reliable results.

Several attempts have been made to introduce reliable methods for the detection of brucellosis. Molecular detection of Brucella melitensis by PCR and real-time PCR has been used in some studies (Alamian et al., 2017; Shakerian et al., 2016). In these methods, specific primer pairs are used for amplification of a specific part of brucella DNA. (Alamian et al., 2017), introduced a novel PCR assay for detecting Brucella bacteria. They found that PCR results were the same as the bacteriological method for Brucella detection (Alamian et al., 2017). However, However, PCR-based methods require high technology laboratories and instruments and Trained personnel that limits these methods (Bayramoglu et al., 2019). DNA probes have also been used for the detection of brucellosis. Surface plasmon resonance is used to detect Brucella melitensis based on the screening of its complementary DNA target using two different designed DNA probes. Although the detection time for the DNA target with two immobilized DNA probes by SPR was found to be short, the interaction between DNA targets and probe 2 was less effective than that of probe 1 (Sikarwar et al., 2017).

Yang et al. developed a label-free amperometric immunosensor for the detection of the Brucella-positive standard serums based on the Brucella melitensis immobilization on the surface of a cysteamine/glutaraldehyde modified screen-standard antibodies, and a linear relationship between the peak current and antibody concentration was reported. Furthermore, the method was found to be capable of detecting small concentrations of anti brucella antibodies. However, they did not test the method on actual serum samples, and how to use this method, in reality, must still be studied further (Yang et al., 2019).

\section{Conclusion}

The majority of available laboratory assays for diagnosis of Brucellosis are qualitative or semi-qualitative. These assays are often time-consuming and require skilled and trained laboratory personnel. Additionally, they are susceptible to optical errors. Other techniques, such as ELISA, require long assay time and advanced laboratory instruments and cannot accurately quantify the antibody levels, which limits their application in all laboratories (Padilla et al., 2010). In this work, a selective and sensitive anti-Brucella antibody nanobiosensor was constructed based on the reduction of the LSPR absorption peak of the modified gold nanoparticles. Our designed biosensor with good analytical performance was successfully applied to determine positive, negative, and all the sera samples. This method delivers not only reproducible results in a shorter time but also requires conventional centrifuges and a simple spectrophotometer. Thus, it could be applied in a clinical diagnostic setting to detect brucellosis as the first LSPR-based nanobiosensor assay.

\section{ACKNOWLEDGEMENTS}

This work was supported by grants from Kerman University of Medical Sciences. The authors appreciate the support of the dissertation grant.

\section{References}

Alamian, S.; Esmaelizad, M.; Zahraei, T.; Etemadi, A.; Mohammadi, M. et al. (2017). A novel PCR assay for detecting brucella abortus and brucella melitensis. osong public health and research perspectives, 8, 65 .

Almuneef, M.; Memish, Z.; Balkhy, H.; Alotaibi, B.; Algoda, S. et al. (2004). Importance of screening household members of acute brucellosis cases in endemic areas. Epidemiology and infection, 132, 533-540. 
Aqeel, T.; Greer, H. F. \& Bumajdad, A. (2018). Novel synthesis of crystalline mesoporous tin dioxide doped with nanogold. Kuwait Journal of Science, 45.

Araj, G. F. (2010). Update on laboratory diagnosis of human brucellosis. International journal of antimicrobial agents, 36, S12-S17.

Bayramoglu, G.; Ozalp, V. C.; Oztekin, M. \& Arica, M. Y. (2019). Rapid and label-Free detection of brucella melitensis in milk and milk products using an aptasensor. Talanta, 200, 263271.

Bellan, L. M.; Wu, D. \& Langer, R. S. (2011). Current trends in nanobiosensor technology. Wiley interdisciplinary reviews: nanomedicine and nanobiotechnology, 3, 229-246.

Cobley, C. M.; Chen, J.; Cho, E. C.; Wang, L.V. \& Xia, Y. (2011). Gold nanostructures: a class of multifunctional materials for biomedical applications. Chemical society reviews, 40, 4456.

Faridli, Z.; Mahani, M.; Torkzadeh- Mahani, M. \& Fasihi, J. (2016). Development of a localized surface plasmon resonance-based gold nanobiosensor for the analytical biochemistry, 495, 32-36.

Fornara, A.; Johansson, P.; Petersson, K.; Gustafsson, S.; Qin, J. et al. (2008). Tailored magnetic nanoparticles for direct and sensitive detection of biomolecules in biological samples. Nano Letters, 8, 3423-3428.

Franco, M. P.; Mulder, M.; Gilman, R. H. \& Smits, H. L. (2007). Human brucellosis. The Lancet infectious diseases, 7, 775-786.

Gomez, M. C.; Nieto, J. A.; Rosa, C.; Geijo, P.; Escribano, M. A. et al. (2008). Evaluation o $\mathrm{f}$ seven tests for diagnosis of human brucellosis in an area where the disease is endemic. Clinical and vaccine immunology, 15, 10311033.

Gu, H.; Xu, K.; Xu, C. \& Xu, B. (2006). Biofunctional magnetic nanoparticles for protein separation and pathogen detection. Chemical Communications, 941-949.

Holzinger, M.; Le Goff, A. \& Cosnier, S. (2014). Nanomaterials for Biosensing applications: a Review. Frontiers in chemistry, 2, 63.

Hong, Y.; Huh, Y.-M.; Yoon, D. S. \& Yang, J. (2012). Nanobiosensors based on localized surface plasmon resonance for biomarker detection. Journal of Nanomaterials, 2012, 111.

Jay, M.; Corde, Y.; Al Lawati, H.; Bose, S.; Al Hamrashdi, A. et al. (2018). Serological, cultural, and molecular evidence of Brucella melitensis infection in goats in Al Jabal Al Akhdar, Sultanate of Oman. Veterinary medicine and science, 4(3):190-205.

Kaden, R.; Ferrari, S.; Alm, E. \& Wahab, T. (2017). A novel real-time PCR assay for specific detection of brucella melitensis. BMC infectious diseases, 17, 230.

Kattar, M. M.; Zalloua, P. A.; Araj, G. F.; Samaha-Kfoury, J.; Shbaklo, H. et al. (2007). Development and Evaluation of realtime polymerase chain reaction assays on whole blood and paraffin-embedded tissues for rapid diagnosis of human brucellosis. Diagnostic microbiology and infectious disease, 59, 23-32.

Leng, W.; Pati, P. \& Vikesland, P. J. (2015). Room temperature seed-mediated growth of gold nanoparticles: mechanistic investigations and life cycle assesment. Environmental science: nano, 2, 440-453. 
Martin, M. N.; Basham, J. I.; Chando, P. \& Eah, S.-K. (2010). Charged gold nanoparticles in non-polar solvents: 10-min synthesis and 2d self-assembly. Langmuir, 26, 7410-7417.

Mirabi, A.; Rad, A. S. \& Ahmadizadeh, S. (2019). Graphene oxide enhances SBA15 ability towards preconcentration as well as determination of barbiturate drug in real samples Kuwait Journal of Science, 46.

Mitka, S.; Anetakis, C.; Souliou, E.; Diza, E. \& Kansouzidou, A. (2007). Evaluation of different PCR assays for early detection of acute and relapsing brucellosis in humans in comparison with conventional methods. Journal of Clinical Microbiology, 45, 12111218 .

Moreno, E.; Pitt, M.; Jones, L.; Schurig, G. \& Berman, D. (1979). Purification and characterization of smooth and rough lipopolysaccharides from brucella abortus. Journal of Bacteriology, 138, 361-369.

Mortazavi, D.; Kouzani, A. Z.; Kaynak, A. \& Duan, W. (2012). Developing LSPR design guidelines. Progress in electromagnetics research, 126, 203-235.

Nielsen, K. \& Yu, W. (2010). Serological diagnosis of brucellosis. Prilozi, 31, 65-89.

Padilla Poester, F.; Nielsen, K.; Ernesto Samartino, L. \& Ling Yu, W. (2010). Diagnosis of brucellosis. The open veterinary science journal, 4.

Salahvarzi, A.; Mahani, M.; TorkzadehMahani, M. \& Alizadeh, R. (2017). Localized surface plasmon resonance-based gold nanobiosensor: determination of thyroidstimulating hormone. Analytical Biochemistry, 516, $1-5$.
Sepulveda, B.; Angelome, P. C.; Lechuga, L. M. \& Liz-Marzan, L. M. (2009). LSPRbased nanobiosensors. Nano today, 4, 244251.

Shakerian, A.; Deo, P.; Rahimi, E.; Shahjavan, A.-R. \& Khamesipour, F. (2016). Molecular detection of brucella melitensis in sheep and goat milk in Iran. Tropical Journal of pharmaceutical research, 15, 913-918.

Sikarwar, B.; Singh, V. V.; Sharma, P. K.; Kumar, A.; Thavaselvam, D. et al. (2017). DNA-probe-target interaction-based detection of brucella melitensis. by using surface plasmon resonance. Biosensors and bioelectronics, 87, 964-969

Yang, W.; Zhang, Z.; Song, H.; Han, X. \& Zuo, Y. (2019). Label-Free amperometric immunosensor for quantitative detection of low-concentration brucella-positive standard serum. Sensors and Materials, 31, 661-671.

$\begin{array}{lr}\text { Submitted: } & 06 / 11 / 2019 \\ \text { Revised: } & 03 / 04 / 2020 \\ \text { Accepted: } & 07 / 05 / 2020 \\ \text { DOI: } & 10.48129 / \text { kjs.v48i3.8581 }\end{array}$

